\title{
TERAPIA OCUPACIONAL EN INCLUSIÓN LABORAL: EXPERIENCIAS A NIVEL LOCAL
}

\author{
OCCUPATIONAL THERAPY IN WORK INCLUSION: LOCAL EXPERIENCES
}

\section{Jacinta Bravo M., Valentina Chávez Q., David Cid P., Rocío Montecino M. ${ }^{1}$, Ximena Toro V. ${ }^{2}$, Rodrigo Sepúlveda P. $^{3}$}

\begin{abstract}
Resumen
El presente estudio exploratorio tiene como objetivo analizar la implementación de dos proyectos de SENADIS para la inclusión laboral de personas con discapacidad (CCR Integral y Proyecto Piloto de Profesionales de Apoyo) en base al relato de Terapeutas Ocupacionales que ejecutaron dichos proyectos en los municipios de Conchalí, Huechuraba, Independencia, La Pintana y Peñalolén entre los años 2011-2013, contribuyendo a la generación de conocimiento en lo que respecta a políticas públicas de inclusión laboral para personas con discapacidad. Este estudio es de tipo cualitativo y está diseñado en base a la Teoría Fundamentada. La recolección de datos se realizó a través del diseño de una entrevista semi-estructurada a las Terapeutas Ocupacionales ejecutoras y a los encargados de dichos proyectos en SENADIS. Las entrevistas abarcaron desde aspectos operativos de la ejecución de los proyectos, hasta reflexiones personales en torno a la experiencia. Se identifican como aspectos significativos las siguientes categorías: proceso de implementación de los proyectos en los Municipios, constitución del cargo de las Terapeutas Ocupacionales, equipo de trabajo, factores que facilitaron la implementación de los proyectos, factores que dificultaron la implementación de los proyectos y reflexión de las Terapeutas Ocupacionales acerca de la experiencia. La presente investigación se centra en conocer el proceso de implementación de políticas públicas de inclusión laboral a nivel municipal, a partir de la experiencia de Terapeutas Ocupacionales ejecutoras de los proyectos ejecutados.
\end{abstract}

\section{Palabras clave:}

Inclusión laboral, Terapia Ocupacional, intervención local, discapacidad.

\begin{abstract}
Abstrac
The aim of this exploratory study is to analyze the execution of two projects proposed by SENADIS for work inclusion, based on the Occupational Therapists report who participated in the execution of both projects in the local councils of: Conchali, Huechuraba, Independencia, La Pintana and Peñalolén between 2011 and 2013, promoting the knowledge generation related to public policies of work inclusion. This qualitative study is based on Founded Theory. The data recollection was done through semi-structured interviews to the Occupational Therapists and two SENADIS professionals in charge of both projects. The interviews included operative aspects like the execution of both projects and also personal thoughts about the experience. The next categories were considered meaningful for the study: The interviewed, execution process of both projects in local councils, Occupational Therapist's position in both projects, work team, factors that collaborated in the execution, factors that interrupted the execution and personal thoughts about the experience. This study is to recognize the execution process of public policies in work inclusion, generating discussions from the Occupational Therapists experience that executed both projects.
\end{abstract}

\section{Keywords:}

Work inclusion, Occupational Therapy, local intervention, disability.

1 Licenciado en Ciencias de la Ocupación Humana, Universidad de Chile.

2 Terapeuta Ocupacional, Universidad de Chile. Master en Integración de Personas con Discapacidad, Universidad de Salamanca. Instructor, Escuela de Terapia Ocupacional, Universidad de Chile.

3 Antropólogo, Universidad de Chile. Magíster en Psicología, Universidad Diego Portales. Doctor (c) en Estudios Latinoamericanos, Profesor Asistente Escuela de Terapia Ocupacional, Universidad de Chile. 


\section{INTRODUCCIÓN}

Durante las últimas décadas, diversos organismos internacionales como la Organización Internacional del Trabajo (1983) y la Organización de las Naciones Unidas (2006), y organizaciones de la sociedad civil han desarrollado convenciones, convenios y recomendaciones enfocadas a la inclusión social de las personas con discapacidad. Debido a la naturaleza multidimensional del concepto de inclusión y su desarrollo interdisciplinar, es que ha resultado difícil Ilegar a consensos en relación a sus fundamentos. Es por esto que diferentes organismos gubernamentales y organizaciones han establecido definiciones operacionales para comprenderlo. Para la comprensión de este estudio, el concepto de Inclusión se entenderá como: "La generación de igualdad de oportunidades y apoyos para los integrantes de un grupo, comunidad o población bajo la idea del respeto a los derechos humanos. Su finalidad debe ser la participación plena de los individuos en la vida económica, comunitaria y cívica, como también en el desarrollo de sus propios potenciales." (Whiteford, G. \& Hocking, C., 2012).

Dentro de la participación en la vida económica, la actividad productiva se puede visualizar como una vía de autorrealización y adquisición de autonomía del individuo (OIT, 1999). La OIT, ha desarrollado desde hace ya tres décadas lineamientos internacionales que abarcan principios y normas relacionadas con el trabajo y las personas con discapacidad, poniendo énfasis en que el objetivo de esta organización es el "Trabajo Decente" para todas las personas, incluidas las personas con discapacidad (OIT, 1999).

En el marco de la conceptualización del término Inclusión señalado, para efectos del presente estudio se propone una definición de Inclusión Laboral a partir del concepto de inclusión junto a la definición de Trabajo Decente propuesta por la Organización Internacional del Trabajo (OIT, 1999). Por lo tanto, el concepto de Inclusión Laboral se entiende como: "la generación igualitaria de oportunidades para los individuos, grupos o comunidades al momento de optar a un trabajo, el cual otorgue un ingreso digno, seguridad y protección social, posibilidad de participación y organización en un contexto de justicia e igualdad de género".

Por su parte en el año 2006 la Organización de las Naciones Unidas, ONU, promulga la Convención de los Derechos Humanos de las Personas con Discapacidad, donde se establece en su artículo 27 "el derecho de las personas con discapacidad al trabajo en igualdad de condiciones que los demás, en ambientes inclusivos, abiertos y accesibles para las Personas con discapacidad", Convención a la que el Estado de Chile adhiere en agosto de 2008.

Como Estado Miembro de la ONU y la OIT, respondiendo a las necesidades visualizadas y cumpliendo con estándares y compromisos a nivel internacional, Chile promulga el año 2011 la Ley No 20.422, que establece "Normas sobre igualdad de oportunidades e inclusión social de personas con discapacidad" (Ministerio de Planificación, Gobierno de Chile. Febrero de 2010). Esta normativa, en el artículo 43 establece que "el Estado, a través de los organismos competentes, promoverá y aplicará medidas de acción positivas para fomentar la inclusión y no discriminación laboral de las personas con discapacidad".

Cumpliendo con la normativa internacional y por mandato de la Ley $N^{\circ} 20.422$, se forma el Servicio Nacional de la Discapacidad, SENADIS, que tiene por finalidad "promover la igualdad de oportunidades, inclusión social, participación y accesibilidad de las personas con discapacidad" (SENADIS, 2013), lo que se traduce, entre otros, en la generación de planes y programas relacionados a la inclusión laboral de personas con discapacidad.

El Fondo Nacional de la Discapacidad (FONADIS, actualmente SENADIS), en el año 2002 implementa el Programa de Intermediación Laboral, PIL, con el objetivo de promover la inclusión laboral de Personas con Discapacidad, que tiene como propósito "Facilitar el acceso al mercado laboral de las personas con discapacidad en igualdad de condiciones. Entregando servicios en el campo de la Intermediación Laboral entre el trabajador/a con discapacidad y el empleo, es decir, acercar la demanda laboral de las personas con discapacidad a la oferta de empresas del sector privado y público" (SENADIS, 2011).

EI PIL fue el primer programa en realizar procesos de intermediación laboral como política pública, implementando sus acciones hasta el año 2012 a través de las oficinas regionales de SENADIS en el país. El programa se finiquita el año 2012.

En este marco se produce un ajuste en el modo de implementación de esta política y se inicia una progresiva descentralización trasladando la responsabilidad del proceso de inclusión laboral de personas con discapacidad a los municipios. En este nuevo escenario los Departamentos de Inclusión Laboral y de Salud de SENADIS Nacional diseñaron dos proyectos pilotos 
orientados a generar oportunidades de empleo para las personas con discapacidad implementándolo a través de las Oficinas Municipales de Intermediación Laboral (OMIL) y los CCR Integral en las comunas seleccionadas para la ejecución de los proyectos pilotos.

Estos proyectos son:

a) Proyecto Piloto de Profesionales de Apoyo (en adelante PP), $y$

b) CCR Integral (en adelante CCRI).

El Proyecto Piloto de Profesionales de Apoyo emerge como una iniciativa liderada desde el Departamento de Inclusión Laboral de SENADIS y financiada por el Departamento de Tecnología Asistiva y Servicios de Apoyo de la misma institución. Consiste en un convenio de transferencia de fondos desde SENADIS hacia los municipios seleccionados, para la contratación de un profesional con experiencia en temas de inclusión laboral, el cual apoye este proceso a través de las OMIL. Otro de los objetivos planteados por el proyecto dice relación con incluir la temática de la discapacidad en los municipios seleccionados. La duración de este proyecto es de seis meses y los municipios en los que se ha llevado a cabo han sido Huechuraba, Conchalí, Estación Central, Peñalolén, Rancagua y Maipú. (Entrevista realizada a Mónica Sotomayor, Departamento de Trabajo, SENADIS, 2013).

El proyecto CCR Integral surge desde el Área de Rehabilitación del Departamento de Salud de SENADIS con el propósito de dar continuidad a la rehabilitación de las y los usuarios/as de los Centros Comunitarios de Rehabilitación (CCR), favoreciendo finalmente su proceso de inclusión laboral desde las OMIL. Estas iniciativas se gestionan a través de un convenio de transferencia de fondos para incluir la temática de la discapacidad en las OMIL, mediante la contratación de una Terapeuta Ocupacional. Además, establece una base de trabajo en red dentro de la comuna para dar respuesta a las necesidades de las personas con discapacidad. Su duración es de un año y ha sido implementado en las comunas de La Pintana, Independencia, Chimbarongo y Concepción. (Entrevista realizada a Miguel Ángel Figueroa, Departamento de Salud, SENADIS, 2013.)

Ambos proyectos son las primeras experiencias concretas a nivel municipal que se implementan a partir de la Ley $N^{\circ} 20.422$, y lo que esta propone en términos de inclusión laboral. Además intentan dar respuesta a las necesidades detectadas por SENADIS de descentralizar el proceso de intermediación laboral. Es fundamental conocer la experiencia de los profesionales que ejecutaron los proyectos PP y CCRI, para comprender la puesta en marcha de la política pública de inclusión laboral a nivel local.

En la actualidad no existe un mayor desarrollo teórico que surja desde la Terapia Ocupacional en relación a temáticas de inclusión laboral a nivel territorial-municipal. Surge de este modo la necesidad de investigar al respecto y conocer la percepción que tienen terapeutas ocupacionales acerca de la implementación de estos proyectos a partir de las experiencias vivenciadas y relatadas por terapeutas responsables de la ejecución de ambos proyectos.

El estudio busca dar respuesta a la siguiente pregunta: ¿Cómo se está llevando a cabo la implementación de proyectos de inclusión laboral (PP y CCRI) ejecutados por Terapeutas Ocupacionales a nivel municipal?

\section{MÉTODO}

El estudio tiene los siguientes objetivos:

a) Conocer el modo en que se ejecutaron los proyectos pilotos PP y CCRI.

b) Conocer las percepciones de terapeutas ocupacionales responsables de la ejecución de ambos proyectos, respecto de implementación, particularmente los referidos a la gestión institucional.

\section{Tipo de metodología}

Para el logro de los objetivos se utilizó una metodología de tipo cualitativo, específicamente la Teoría Fundamentada (Hernández, S., 2004), cuyo propósito es el descubrir conceptos, hipótesis y relaciones a partir de los datos, de manera de producir teorías de carácter emergente para la comprensión y explicación de los fenómenos. El método de la Teoría Fundamentada se conoce como Comparativo Constante (Hernández, N., 2007), ya que alude a una revisión y comparación de los datos de manera continua y sistemática para poder ir construyendo teoría (Sandoval, C., 1996).

Además, el estudio es exploratorio, el cual tiene como finalidad "aumentar el grado de familiaridad con fenómenos relativamente desconocidos, obtener información sobre la posibilidad de llevar a cabo una investigación más completa sobre un contexto particular de la vida real, investigar problemas del comportamiento humano que consideren cruciales los profesionales de determinada área, identificar conceptos o variables 
promisorias, establecer prioridades para investigaciones posteriores o sugerir afirmaciones (postulados) verificables" (Dankhe, G. L., 1989).

Las entrevistadas corresponden a cuatro Terapeutas Ocupacionales que se desempeñaron en cinco municipios distintos, tanto en el CCRI como en el PP.

Terapeuta Ocupacional 1 egresa el año 2011 de la Universidad de Chile, siendo su primera experiencia laboral la participación en el PP en la Municipalidad de Independencia. Anteriormente había realizado prácticas relacionadas con temas de inclusión laboral y generación de políticas públicas. Esta profesional fundamenta su intervención a través de un enfoque de Derechos Humanos. Éste se plasma en la visión que refiere de su concepto de Inclusión, el que consistiría en igualdad de acceso al trabajo y servicios para todas las personas, siendo el ambiente el que debe modificarse en función de los/as usuarios/as.

Terapeuta Ocupacional 2, es profesional desde el año 2009 cuando egresa de la Universidad de Chile. Se desempeña en diversos espacios desde el área pública y el año 2010 ingresa a la Oficina de Discapacidad de la Municipalidad de Huechuraba, donde posteriormente, en el año 2013, sería la encargada de implementar el PP. Ella comprende las problemáticas de los/as usuarios/as a través del Modelo de Ocupación Humana, entendiendo que la inclusión se logra a través de la adquisición del rol de trabajador, donde la persona con discapacidad se transforma en un ente productivo para la sociedad.

Terapeuta Ocupacional 3 egresa el año 2011 de la Universidad Nacional Andrés Bello. Se desempeña en diversos cargos relacionados a la inclusión laboral. El 2012 forma parte del proyecto CCRI en la Municipalidad de La Pintana por un periodo de 6 meses, dejando el cargo para posteriormente comenzar a trabajar en la Municipalidad de Peñalolén, encargándose del PP de dicha comuna. Su visión del concepto de Inclusión está relacionada con la perspectiva de Derechos Humanos, destaca que todas las personas puedan escoger un puesto de trabajo en igualdad de condiciones y oportunidades. Los modelos que refiere fundamentan su intervención son el Modelo de Ocupación Humana y de Redes.

Terapeuta Ocupacional 4 egresa el año 2010 de la Universidad Autónoma de Chile. Se desempeña en la Asociación Chilena de Seguridad (ACHS), ligada al área de ergonomía. Luego es contratada para desempeñarse como ejecutora del PP en la Municipalidad de Conchalí. Esta profesional posee una visión ligada al Modelo de Integración, en donde la persona es quien debiese adquirir las competencias necesarias desarrollando habilidades y hábitos laborales para adaptarse a las exigencias del medio.

Para la recolección de datos se utilizó una entrevista semi-estructurada con tópicos centrales establecidos previamente en relación a lo considerado relevante para la investigación. De acuerdo a esto, surgieron 26 tópicos agrupados en el ejercicio profesional de las Terapeutas Ocupacionales, caracterización de la comuna, visión de las Terapeutas Ocupacionales acerca de los conceptos de trabajo, inclusión y discapacidad, acciones de las profesionales (evaluación, contacto con empresas, colocación, difusión, trabajo en red, entre otros) constitución del equipo de trabajo y apreciación de este, enfoques y modelos utilizados por las profesionales, caracterización del proceso previo a la implementación, posibilidad de continuidad del proyecto, relaciones con SENADIS, apreciación y reflexión con respecto al proyecto y la labor realizada, facilitadores y barreras para la implementación.

Las entrevistas fueron desarrolladas entre los meses de junio y agosto de 2013, y contaron con una duración aproximada de una hora y 30 minutos. Todas las entrevistas fueron grabadas por los investigadores y posteriormente transcritas. Se utilizó un diseño emergente para codificar cada pregunta de forma abierta y finalmente ser analizada.

La selección de los participantes se llevó a cabo bajo los siguientes criterios de inclusión:

- Que los/las entrevistados/as participaran de los proyectos implementados por SENADIS (PP y/o CCRI).

- Que los/as entrevistados/as fueran Terapeutas Ocupacionales.

- Que los/las entrevistados/as se encontraran o hayan trabajado en la OMIL y/u Oficina de la Discapacidad de la municipalidad de implementación de los proyectos.

- Que los/las entrevistados/as hayan participado en la ejecución de proyectos dentro de la Región Metropolitana.

- Que los/las entrevistados/as confirmaran su participación dentro de los plazos establecidos.

Las entrevistadas fueron contactadas a través de correos electrónicos y/o contacto telefónico, solici- 
tando su colaboración en la investigación. A medida que fueron contactadas se les explicó los objetivos de la investigación, y la importancia e implicancias de su participación. Si accedían a participar se planificaba un encuentro posterior para realizar la entrevista. Dicha entrevista fue realizada en algún lugar que fuera cómodo para la entrevistada, ya sea en su lugar de trabajo o en un café. El total de entrevistadas fue de cuatro Terapeutas Ocupacionales, de las cuales dos pertenecían al proyecto CCR Integral, específicamente de las comunas de Independencia y La Pintana, y otras dos Terapeutas Ocupacionales correspondientes al Proyecto Piloto de las comunas de Conchalí, Huechuraba y Peñalolén, considerando que una de las entrevistadas trabajó en dos de los proyectos (La Pintana y Peñalolén).

El encuadre de la entrevista consideró explicitar nuevamente los objetivos y la finalidad de la investigación, las implicancias de su participación, temas y preguntas a realizar. Posteriormente se informó sobre la grabación de la entrevista y se realizó el consentimiento informado y su libre asentimiento registrado en la grabación.

Además, se realizó una entrevista semi-estructurada con dos profesionales, uno del Departamento de Inclusión Laboral y otro del Área de Rehabilitación del Departamento de Salud, que eran los encargados de la planificación y diseño de dichos proyectos respectivamente. La entrevista fue gestionada de igual manera que la realizada con las Terapeutas Ocupacionales.

Finalmente las entrevistas fueron transcritas y codificadas de acuerdo a tópicos relevantes que emergían durante el análisis.

\section{Aspectos Bıó́ticos}

La participación de las Terapeutas Ocupacionales en esta investigación no significó riesgo físico, psíquico o moral alguno. Todas las participantes fueron informadas, mediante un consentimiento informado de palabra y registrado en una grabación, oportunamente y previo a la entrevista, sobre las implicancias de este estudio y que podían negarse a continuar y/o abandonar el proceso de la investigación sin perjuicio alguno en el momento en el que ellas lo estimaran necesario. Los propósitos del consentimiento informado fueron verificar la comprensión de la información entregada en relación a los objetivos del estudio, las características de su participación, explicitar nuevamente su libertad de participación y autorizar al equipo de investigadores para utilizar la información entregada con fines académicos y científicos resguardando su confidencialidad.

Los principios bioéticos de este estudio se relacionan esencialmente con los principios de justicia y beneficencia, en la medida que busca generar información y reflexiones que puedan aportar para el diseño e implementación de nuevos programas que finalmente colaboren en la toma de decisiones para disminuir situaciones de desigualdad y beneficiar tanto a los y las profesionales que se desempeñen en proyectos pilotos de SENADIS, como a las personas con discapacidad usuarias/os de los programas de intermediación laboral, avanzando hacia dar respuesta a sus necesidades de manera respetuosa, justa y respetando sus derechos. Los principios de autonomía se reflejan en la elaboración del consentimiento informado y el principio de no maleficencia al buscar que este estudio no produzca daño alguno.

\section{Resultados}

A partir del análisis de los tópicos incluidos en las entrevistas, se obtuvo información sobre la experiencia de las entrevistadas acerca de la implementación de los proyectos CCRI y PP. Estos resultados se agrupan en siete categorías presentadas a continuación:

\section{1) Proceso de implementación de los proyectos en los Municipios}

De acuerdo al relato de las entrevistadas, al inicio de la implementación de los proyectos, se detectaron una serie de conflictos e irregularidades a nivel administrativo y operacional, causados en su gran mayoría por procesos eleccionarios a nivel municipal.

"Lo que pasa también en el convenio (...) se articularon mal las fechas o desde acá se vio mal el asunto." Además "esto debería haber funcionado desde octubre del año pasado, pero por distintos motivos, la administración de acá también cambió y hay un problema que anotaron mal el número de cuenta y todo, entonces no podía empezar a funcionar." 
(Terapeuta Ocupacional 1). "iEso fue del terror! Yo no pude iniciar en la fecha que correspondía porque estaban en cambio de alcalde." (Terapeuta Ocupacional 4).

Es por esto que se producen dificultades para implementar los proyectos en las fechas correspondientes, generando así un estancamiento en el flujo de usuarios/ as beneficiados/as.

Además, es importante destacar que no todos los proyectos fueron ejecutados desde las OMIL, siendo este el caso de Huechuraba, el cual se ejecutó desde la Oficina de la Discapacidad.

"En algunos municipios nos costó bastante. Por ejemplo en Huechuraba nos costó especialmente y no pudimos poner este proyecto piloto dentro de la OMIL. Tuvimos que ponerlo en la Oficina de Discapacidad... no había un interés de parte de la OMIL de ser parte de este proceso" (Encargada Departamento de Trabajo. SENADIS).

Esto significó que el proyecto no se ejecutara desde el organismo municipal correspondiente (OMIL) para el proceso de intermediación laboral, por lo que las personas con discapacidad no poseen igualdad de oportunidades para optar y acceder a los servicios otorgados por la municipalidad al igual que el resto de la comunidad.

\section{2) Rol de las Terapeutas Ocupacionales en el cargo}

Se puede apreciar que la labor de las Terapeutas Ocupacionales, a pesar de utilizar enfoques y modelos diversos para sustentar su intervención, consistió en contactarse con las empresas, entrevistar y evaluar usuarios, analizar puestos de trabajo, sensibilizar a las empresas, colocar a los beneficiarios y, en algunos casos, realizar tareas de apresto laboral. A pesar de que no estaba contemplado en el proyecto, debieron realizar además difusión dentro del municipio y a los habitantes de la comuna respecto del proyecto.

Terapeuta Ocupacional 1 refiere que, con anterioridad a la puesta en marcha del proyecto, debió realizar difusión del mismo al interior del municipio, dado que no se abordaban temáticas de discapacidad en sus organismos. En relación al proceso de intervención con los/as usuarios/as, las evaluaciones abarcaban datos generales de la persona y aspectos propios de la disci- plina dando énfasis a las Actividades de la Vida Diaria (AVD). Además, realizaba actividades de apresto laboral mediante la estrategia de role playing. Se encargaba de contactar empresas para generar posibles puestos de trabajo y una vez colocado el usuario, se preocupaba del seguimiento de este, idealmente por al menos seis meses, respaldando tanto al usuario como a la empresa en el proceso.

La Terapeuta Ocupacional 2 trató de diversificar su acción, no sólo prestando servicio a las personas sino que también a las empresas con la finalidad de abrir más puestos de trabajo y consolidar la alianza con éstas.

\section{"Entonces yo dije: si trabajo las empresas y a estas les doy seguridad y me abren un puesto, es muy probable que si yo estoy ahí y la empresa siente confianza me abrirá puestos distintos o más puestos" (Terapeuta Ocupacional 2).}

De acuerdo a esto y con el fin de responder a la confianza de las empresas, realizaba trabajo en red para colocar personas que, aunque no fuesen sus usuarios/ as de su proyecto, pudiesen cumplir con el perfil y los requerimientos de los puestos de trabajo ofertados por las empresas. La evaluación que realizaba consistía en una entrevista inicial y en la aplicación de evaluaciones basadas en la medición de habilidades motoras, cognitivas y sociales a lo largo de varias entrevistas para asegurar adherencia al proceso.

En relación a la Terapeuta Ocupacional 3, una de las acciones realizadas fue la conformación de una red de Terapeutas Ocupacionales de diversos organismos de salud de la comuna de Peñalolén (CCR, COSAM, CESFAM, INR PAC, entre otros) para apoyar y hacer más eficiente el trabajo de selección, colocación y seguimiento de los/as usuarios/as.

"En el caso de que yo no pueda realizar el análisis de puesto y seguimiento, voy a recurrir a este Terapeuta Ocupacional para que él pueda realizar lo posible durante el proceso con el usuario. Entonces la idea es que cada profesional se haga cargo, pero yo ya haber concientizado a la empresa en esta materia (...) el Terapeuta Ocupacional puede realizar también continuidad de tratamiento con su usuario y realizar el 
seguimiento telefónico o en terreno según corresponda." (Terapeuta Ocupacional $3)$.

Durante el proceso de evaluación, la Terapeuta Ocupacional 3 determinaba el tipo de trabajo más pertinente para el usuario (dependiente o independiente) y realizaba un taller de apresto laboral grupal abordando temáticas relacionadas al trabajo. Además su intervención se basaba en sensibilización a las empresas y a los compañeros de trabajo, como también a la colocación laboral.

La Terapeuta Ocupacional 4, se encargó de contactar empresas para abrir nuevos puestos de trabajo, concientizando por medio de los principios de la Responsabilidad Social Empresarial. Luego realizaba las colocaciones y el posterior seguimiento.

"No les mencioné mucho capacitación porque más que haber alguien encargado de capacitación, se derivaba a las ONG, hacer redes intersectoriales." (Terapeuta Ocupacional 4).

Con esto se hace referencia a que las capacitaciones o actividades de apresto laboral eran realizadas por otros organismos y no por la Terapeuta Ocupacional. La difusión sobre el programa se realizó a través de la Dirección de Desarrollo Comunitario (DIDECO) que llevó a cabo capacitaciones a los y las funcionarios/as de la OMIL en temáticas de discapacidad.

Cabe destacar que, más allá de las acciones propias de intermediación laboral, cada una de las Terapeutas Ocupacionales llevó a cabo diversas labores de acuerdo a las necesidades que visualizó en su comunidad y a la propia comprensión del proceso y de los proyectos en sí.

\section{3) Equipo de trabajo}

Las Terapeutas Ocupacionales debieron integrarse a los organismos municipales respectivos, con equipos de trabajo ya consolidados. Estos equipos eran diversos en su conformación siendo distintos en cada municipio.

La Terapeuta Ocupacional 1 se incorporó a un equipo de trabajo que contaba con Administradores Públicos y una Psicóloga Laboral, los que no contaban con experiencia con personas con discapacidad, pero demostraron interés en colaborar y aprender con el proyecto.

La Terapeuta Ocupacional 2 se desempeña de manera independiente desde la Oficina de Discapacidad, que contaba con un equipo conformado por una Asistente
Social y dos Kinesiólogos. Además una Educadora Diferencial y un monitor de Jaws apoyaban de manera externa y voluntaria las labores de la Terapeuta Ocupacional.

La Terapeuta Ocupacional 3 se incorporó en ambos proyectos a las OMIL de las municipalidades correspondientes.

"Allá (La Pintana) trabajaba con un Psicólogo que sinceramente no fue una ayuda en todo el proceso. Él tenía una visión muy distinta, era Psicólogo clínico. Acá (Peñalolén) yo estoy sola. Si necesito un apoyo en general voy y pregunto quién me apoya, pero es una cosa de voluntades." (Terapeuta Ocupacional 3).

La Terapeuta Ocupacional 4 refiere: "Yo trabajaba directamente con las personas encargadas de la OMIL, que eran el encargado de colocaciones y el encargado de fomento productivo."

Todas las Terapeutas Ocupacionales, exceptuando el caso de la Terapeuta Ocupacional 2, se incorporaron a equipos en donde sus integrantes no manejaban la temática de discapacidad, por lo tanto, además de intermediar, debieron capacitar y sensibilizar al equipo de trabajo en temas de discapacidad, salud e inclusión laboral.

\section{4) Factores que facilitaron la implementación y desarrollo de los proyectos}

La Terapeuta Ocupacional 1 refiere como facilitadores del desarrollo del proyecto el apoyo e involucramiento de autoridades municipales a la Red Incluye y el reconocimiento de la labor de la profesional dentro del equipo de trabajo de la OMIL.

"Partiendo que el alcalde se fuera a sumar a esto. Es el único alcalde de la Red Incluye. (...) Entonces el alcalde con su nueva forma de ver comunidad y su trabajo muy desde la comunidad, ha permitido tremendamente llevar a cabo el programa" (Terapeuta Ocupacional 1).

Además destaca la formación académica que recibió, como también el apoyo brindado por la Escuela de Terapia Ocupacional de la Universidad de Chile y ser habitante de la comuna de Independencia. 
La Terapeuta Ocupacional 2 reconoce como facilitador la relación generada con las empresas y su positiva experiencia al contactar y generar redes con el sector privado, lo que significó la generación de empleos para personas con discapacidad.

Las Terapeutas Ocupacionales 3 y 4 refieren que la formación académica recibida y el interés en temáticas de trabajo e inclusión fueron fundamentales para facilitar el desarrollo de los proyectos.

\section{5) Factores que dificultaron la implementación de los proyectos}

A partir del relato de las Terapeutas Ocupacionales se detectaron múltiples factores que dificultaron la implementación, ejecución y desarrollo de los proyectos. Particularmente estas dificultades se presentaron tanto en la relación con SENADIS, como en el proceso de implementación y desarrollo de los proyectos en el organismo municipal.

Las principales dificultades relatadas por las profesionales con SENADIS fueron la falta de esclarecimiento en torno a la implementación de los proyectos, sus objetivos, apoyo técnico durante el proceso, así como los criterios establecidos para evaluar las metas y continuidad de las profesionales. Asimismo, la corta duración de los proyectos según sus propias referencias, perjudicaba su desempeño laboral y cumplimiento de los seguimientos de los usuarios colocados y metas.

Las dificultades expuestas no sólo afectaron a las Terapeutas Ocupacionales sino que también a las autoridades y funcionarios municipales:

"Pero que a mí me hayan venido a ver acá y yo que le pueda mostrar mis carpetas y todas mis cosas. Yo no he tenido mayor orientación o que alguien me pregunte "oye, ¿has tenido un problema en tu ejecución?", nada. (...) El mismo hecho de que te digan que no vas a seguir en agosto y que yo tengo seguimientos que hacer, es una barrera, porque esto se va a acabar y se va a acabar no más. Como que esa es mi sensación y la sensación de mi jefatura también, de que SENADIS se tiró y chao, nunca más nos hicimos cargo" (Terapeuta Ocupacional 1).
" $Y$ en un principio no estuvieron fijadas las metas. Nos dijeron (SENADIS) no, miren, estamos probando, no vamos a medir el número de personas colocadas porque sabemos que a veces hay que hacer trabajos muy largos para tener sólo una persona colocada. Así que el foco no va a estar en eso. Pero a la mitad del proyecto sí importaban las personas colocadas (...) Entonces les costó incluso a ellos ponerse de acuerdo en cuáles eran las metas, cómo iban a medir si el programa resultaba o no. También el periodo para municipalidades que no tenían nada previo, seis meses es muy poco". (Terapeuta Ocupacional 2).

"Hubo poca claridad respecto hasta dónde era la labor de SENADIS, si pasado este tiempo iba a continuar o iba a quedar el cargo a disposición del municipio." (Terapeuta Ocupacional 3).

En relación al contexto municipal, se identificaron falencias en cuanto a la relación del organismo municipal desde el cual trabajaron las profesionales con organismos relevantes en la ejecución del proyecto, siendo estos las Oficinas de Discapacidad y las OMIL. Asimismo reconocieron dificultades dentro del equipo de trabajo al cual pertenecían en torno al abordaje de personas con discapacidad en el área de trabajo.

"Faltó comunicación entre los mismos organismos de la comuna, entonces la Oficina de Discapacidad fue súper resistente. Los otros centros de salud nunca quisieron incorporarse como el COSAM. Nunca supe si había algún nexo con el hospital, no había mayor información al respecto. Entonces eso fue dificultoso" (Terapeuta Ocupacional 3).

"Si yo me muevo de acá, de mi puesto de trabajo y llega alguien con discapacidad, mis compañeros no van lo van a atender porque tú eres la de discapacidad y es decir como el tema de discapacidad es de todos" (Terapeuta Ocupacional 1).

Por último mencionar el desinterés que algunas Terapeutas Ocupacionales apreciaron por parte de algunas empresas contactadas para abordar temáticas de 
inclusión laboral y la apertura de puestos de trabajos para los usuarios beneficiados.

"Las cosas difíciles son que tú mandas 100 mails para distintas empresas y te responden cinco. Ya, lograste contactarte con esas cinco. Después y es lo que me ha pasado, he ido a visitar empresas y tengo que mandarle correos todas las semanas para que me digan ¿Cuáles son las vacantes? ¿Cuáles son las vacantes? Tienes que estar constantemente persiguiéndolos, diciéndoles" (Terapeuta Ocupacional 1).

\section{6) Reflexión de las Terapeutas Ocupacionales acerca de la experiencia}

"O sea el programa se medía en base a la intermediación de usuario, a la colocación. Yo te podía decir; en Call Center yo metí dos personas. Pero el proceso de intermediación en la empresa llevó a que ahora tengan 45. Pero ¿cuántas son de Huechuraba? ¿Entienden? ¿cómo lo rindo? En números son dos personas, $o$ sea súper poco. Pero ¿Cómo le pongo que el proceso fue tan grande que la empresa llegó a hacer modificaciones en recursos humanos?" (Terapeuta Ocupacional 2).

De acuerdo a la cita anterior, la profesional manifiesta que la forma de medición del proyecto no se planteó de manera adecuada, debido a que se evaluaba en base a aspectos cuantitativos como el número de colocaciones y no en relación a aspectos cualitativos, como la generación de nuevas comprensiones acerca de discapacidad y trabajo reflejados en los cambios estructurales que llevaron a cabo las empresas.

"Es como que te dejan a la buena de Dios y como que tú tienes que organizarte y todo. Aquí se respeta mucho si viene algo de gobierno central (...) Si ellos mismos hicieran más presencia, si ellos mismos explicaran mucho más el tema al alcalde y a un montón de gente, tiene otra visión, otra forma de ser percibido por ellos y recibido también, como mucho más acogedores al tema. Creo que falta, sé que SENADIS está haciendo mil cosas y lo encuentro súper bueno" (Terapeuta Ocupacional 1).

De colocar a cualquier profesional a hacer este trabajo, tiene que haber una conversación entre SENADIS y la alcaldesa, o alguien que tome decisiones, el director de la OMIL, por último (...) Eso es algo que ellos tienen que considerar y que no hay capacitación. (...). Además nunca supe si existían pautas específicas para cada proceso de intermediación, como no sabía eso, yo creé y realicé mis propias pautas de ingreso y de análisis de puesto de trabajo" (Terapeuta Ocupacional 3).

Lo anteriormente referido expresa la necesidad de organización y comunicación entre el organismo local y el gobierno central para dar a conocer los proyectos y favorecer la posterior ejecución de éstos. Además se suma la situación de que existió una falta de apoyo técnico manifestado por las Terapeutas Ocupacionales; falta de capacitaciones, material de apoyo para realizar el proceso de evaluación y seguimiento de los beneficiarios del proyecto y falta de apoyo en los convenios políticos para generar redes con los organismos pertinentes.

"Por lo menos tendría que durar un año (el proyecto) porque si queremos dejar temáticas de discapacidad al interior del municipio y no sólo de discapacidad, sino que de inclusión social y laboral al interior del municipio, en seis meses no se hace. $O$ sea, no se hace para nada. Si querían hacer algo, tendrían que haber planificado más" (Terapeuta Ocupacional 4).

El desempeño de la Terapeuta Ocupacional en la ejecución del proyecto se vio perjudicado por su corta duración. Ella manifiesta la dificultad que significó en la instauración de la temática de inclusión social a nivel del organismo ejecutor. Por otro lado, la profesional refiere que desde SENADIS no existió una planificación 
y diseño adecuados en relación al cumplimiento de los objetivos de ambos proyectos.

"Lo que sí quiero rescatar es la apuesta de SENADIS por abrir esta puerta. El hecho de explorar poniendo a un Terapeuta Ocupacional en una OMIL, lo encuentro súper bueno porque es desde aquí de donde nace la necesidad de la persona de insertarse en algún puesto de trabajo (...) Fue un buen trabajo desde SENADIS para potenciar, empoderar al Terapeuta Ocupacional dentro de un organismo como la OMIL." (Terapeuta Ocupacional 3).

La profesional refiere una buena relación y comunicación con SENADIS al momento de implementar el proyecto, además destaca la incorporación de un profesional familiarizado en temas de discapacidad a la OMIL. Por otro lado señala que una de las mayores dificultades fue entablar redes de trabajo entre los organismos para trabajar en conjunto en las municipalidades, además de la falta de información, difusión y claridad en cuanto al proyecto.

\section{DisCUSIÓN}

El objetivo general de esta investigación ha sido analizar la implementación de dos proyectos de SENADIS para la inclusión laboral de personas con discapacidad (CCR Integral y Proyecto piloto de Profesionales de Apoyo) en base al relato de Terapeutas Ocupacionales que ejecutaron dos proyectos piloto. A través de estas experiencias fue posible conocer la percepción de las Terapeutas Ocupacionales en relación a la generación y planificación de proyectos a nivel del gobierno central en el área de inclusión laboral, como también acerca de su posterior implementación a nivel local, vinculados a la incorporación al trabajo de personas con discapacidad.

El aporte primario de esta investigación se centró en el conocimiento de la experiencia de Terapeutas Ocupacionales en relación a inclusión laboral de personas con discapacidad, es decir la forma concreta en que esta es llevada a cabo por las profesionales en diversos contextos municipales, así como identificar facilitadores y barreras que están presentes durante la puesta en marcha de los proyectos que buscan dar respuesta a dicho proceso. Asimismo, es posible rescatar que a partir de esta investigación surge un precedente para el diseño e implementación de proyectos futuros, que busquen responder a las necesidades de personas con discapacidad en relación al trabajo.

Los dos proyectos en sí poseen un planteamiento y objetivos de carácter inclusivo, referentes a que la temática de discapacidad sea comprendida y abordada en todos los espacios municipales por los profesionales que se desempeñan en estos, a través de la generación de redes entre el organismo ejecutor y otros organismos municipales con la finalidad de que las personas con discapacidad tengan igualdad de oportunidad de acceso al trabajo. Sin embargo, la puesta en marcha de estos proyectos se desarrolla de manera excluyente, incorporando un profesional externo al municipio, quien se encargó exclusivamente de las temáticas de discapacidad, siendo éste el único responsable de intermediar laboralmente a esta población.

El trabajo de las Terapeutas Ocupacionales estuvo mayormente ligado a colocaciones laborales, respondiendo a la percepción que tenían de los objetivos de los proyectos, lo cuales no fueron planteados con claridad por SENADIS desde un principio, como se explicita en los ítems número cinco y seis de los resultados. Es por esto que la labor de instaurar la temática de discapacidad dentro de las municipalidades fue de carácter secundario y no logrado en su totalidad. Adicionalmente, la forma de medición de resultados se basa en números de colocación laboral. Visto de esta forma y de acuerdo al concepto de Inclusión mencionado en la introducción de esta investigación, es que los proyectos ejecutados en las municipalidades no responden a un proceso inclusivo per se.

Asimismo los equipos de las OMIL, previo y durante la estancia de las profesionales, no fueron preparados para desempeñarse en la intermediación laboral de personas con discapacidad una vez finalizado el convenio, por lo que en la mayoría de los municipios se perdieron las directrices y lineamientos propuestos por las Terapeutas Ocupacionales para abordar este proceso.

SENADIS, por su parte, plantea que la manera más inclusiva de realizar el proceso de intermediación laboral es capacitando al equipo de trabajo de la OMIL y que este sea capaz de realizar este proceso con toda la población, incluidas las personas con discapacidad. Frente a esto y posterior a la implementación de los proyectos surge la interrogante: ¿No será que la incorporación de Terapeutas Ocupacionales a las OMIL re- 
presenta una situación de acción positiva para equiparar oportunidades para las personas con discapacidad?

Por otro lado, el financiamiento por parte de SENADIS para ambos proyectos tenía una duración definida (seis meses el PP y un año el CCRI) por lo que no estaba contemplada la continuidad de los profesionales en las municipalidades y no se consideró para su implementación el que cada municipio se comprometiera a dar continuidad a este proyecto.

Esto se traduce en que si las autoridades eran capaces de visualizar y entender la importancia de la inclusión laboral de personas con discapacidad, como también los resultados y beneficios obtenidos de los proyectos, iban a dar continuidad al trabajo realizado por las Terapeutas Ocupacionales y así instaurar la temática de discapacidad en los espacios municipales. En aquellos municipios en que no se dio continuidad a los proyectos implementados, no se observaron cambios sustanciales en relación al proceso de intermediación laboral de personas con discapacidad.

De acuerdo a lo esperado por SENADIS, las profesionales ejecutoras debían responder a diversas acciones que abarcaban aspectos tan variados como análisis de puesto de trabajo y colocaciones, como también a aspectos que se alejan de la intermediación laboral propiamente tal, referentes a la formación de redes, instauración de la temática de discapacidad tanto en el municipio como en el equipo de trabajo, difusión del proyecto, entre otros.

Gran parte de estas acciones no fueron explicitadas en un comienzo y fueron surgiendo durante el desarrollo de ambos proyectos de acuerdo a las necesidades visualizadas en cada espacio municipal. Esto, sumado al tiempo de duración de los proyectos, dificultó la posibilidad de cumplir con todas ellas en su totalidad.

Si bien los objetivos que planteó SENADIS buscaban generar cambios estructurales y operacionales en el municipio para responder a las problemáticas de las personas con discapacidad en relación al trabajo, el éxito de los proyectos se midió en base a estadísticas, específicamente usando como indicador las colocaciones laborales efectivas, abordando sólo aspectos cuantitativos puntuales al momento de evaluar los proyectos.

Esto se condice con la manera en que se conciben las políticas públicas actualmente en Chile bajo la lógica del modelo económico neoliberal, apuntando a resolver problemáticas sociales a través de la entrega de recursos económicos focalizados y esporádicos, generando así soluciones puntuales a corto plazo, sin cambios sustanciales que logren solucionar la problemática de raíz.

Pese a las dificultades de diseño y gestión de los proyectos pilotos de SENADIS, es necesario valorar que ambos son la primera ventana que se abre a un proceso inclusivo de intermediación laboral que buscaba descentralizar y generar una respuesta local a las problemáticas de las personas con discapacidad en cada municipio. Los logros se plasman en la generación de nuevas formas de trabajo y disposición de los trabajadores de las OMIL, para orientar su accionar hacia un servicio que pueda atender las necesidades laborales de todos los ciudadanos. Además, los resultados del presente estudio pueden contribuir a la construcción de nuevos proyectos y programas por parte de SENADIS en pro de la inclusión laboral de personas con discapacidad.

A modo de conclusión, cabe el cuestionamiento de que si es válido buscar la inclusión desde la exclusión, en este caso desde la acción positiva o basta con tener a un profesional entendido sobre materias de inclusión laboral en una OMIL ¿Debiera una persona con discapacidad necesitar a un Terapeuta Ocupacional para poder ser incluido laboralmente? ¿Los Terapeutas Ocupacionales pueden ser facilitadores válidos para la inclusión laboral de las personas con discapacidad? ¿Está la sociedad preparada para no hacer diferencias entre las personas?

Es posible que la respuesta se encuentre al entender que el tema central no es la búsqueda de la inclusión laboral en sí de las personas, sino que más bien se debe trabajar desde la perspectiva de la exclusión social, realizando una transformación y cambios estructurales a nivel social, político, económico e institucional, que se encaminen hacia una genuina inclusión social. Un cambio de foco permitiría trabajar desde la concepción de ciudadano con un enfoque de derechos, mediante la adopción de perspectivas más amplias que garanticen el acceso equitativo a esos derechos. Es por esto que las políticas públicas relacionadas con la inclusión de personas con discapacidad deben fortalecer la igualdad de oportunidades que disponen los ciudadanos, reconociendo las inequidades y exclusiones que existen actualmente (Sandoval, C., 1996). 


\section{Bibliografía}

Dankhe, G. L. (1989). Investigación y Comunicación, en C. Fernández-Collado y Dankhe G. L. (comps.), La Comunicación Humana: Ciencia Social. México: Mc Graw-Hill.

Extracto de entrevista realizada a Miguel Ángel Figueroa, Departamento de Salud, SENADIS.

Extracto de entrevista realizada a Mónica Sotomayor, Departamento de Trabajo, SENADIS.

Hernández, N. (2007). El Método Comparativo Continuo: Una Alternativa para la Construcción Teórica. Facultad de Ciencias de la Educación, Universidad de Carabobo. Venezuela.

Hernández, S. (2004). Metodología de la Investigación. Mc GrawHill. México.

Ministerio de Planificación, Gobierno de Chile (febrero de 2010). Ley $N^{\circ}$ 20.422, Establece Normas sobre la Igualdad de Oportunidades e Inclusión Social de Personas con Discapacidad. Santiago, Chile.

Noguera, J. (2002). El Concepto de Trabajo y la Teoría Social Crítica. Revistes Catalanes amb Accés Obert, 68, 141-168. Barcelona, España.

Organización de las Naciones Unidas (2006). Convención Sobre los Derechos de las Personas con Discapacidad. Asamblea General de Naciones Unidas (págs. 22-23). Nueva York, Estados Unidos.

Organización Internacional del Trabajo (1983). Convenio 159: Convenio sobre la adaptación y readaptación profesional y el empleo (personas inválidas). Conferencia General de la Organización Internacional del Trabajo. Ginebra, Suiza.
Organización Internacional del Trabajo (1983). Recomendación 168: Recomendación sobre la adaptación y readaptación profesional y el empleo (personas inválidas). Conferencia General de la Organización Internacional del Trabajo. Ginebra, Suiza.

Organización Internacional del Trabajo (1999). Trabajo Decente: Memoria del Director General a la $87^{a}$ reunión de la Conferencia Internacional del Trabajo. Ginebra, Suiza.

Organización Internacional del Trabajo (2013). Estudio: Factores para la Inclusión Laboral de las Personas con Discapacidad. Santiago, Chile.

Organización Panamericana de la Salud (2001). Desafío a la Falta de Equidad en la Salud. De la Ética a la Acción. The Rockefeller Foundation. Washington, D.C, Estados Unidos.

Roberto Hernández Sampieri, C. F. (2004). Metodología de la Investigación. México: Mc Graw-Hill.

Sandoval, C. (1996). Investigación Cualitativa. Instituto Colombiano para el Fomento de la Educación Superior, ICFES. Bogotá, Colombia.

SENADIS (2011). Subsecretariía de Trabajo. Recuperado el 25 de 10 de 2013, de http://www.subtrab.trabajo.gob. cl/wp-content/uploads/downloads/2011/05/ProgramaIntermediaci\%C3\%B3n-Laboral-SENADIS.pdf

SENADIS, (2013). www.senadis.gob.cl. Recuperado el 25 de 10 de 2013, de http://www.senadis.gob.cl/pag/8/352/mision_y_objetivos_estrategicos

Whiteford, G; Hocking, C. (2012). Ciencias de la Ocupación: Sociedad, inclusión, participación. Wiley-blackwell. Pág.: 168. Oxford, Inglaterra. 